\title{
Is postmastectomy radiotherapy really needed in breast cancer patients with many positive axillary lymph nodes?
}

\author{
Tanja Marinko, Karmen Stanic \\ Department for Radiotherapy, Institute of Oncology Ljubljana, Ljubljana, Slovenia
}

Radiol Oncol 2018; 52(3): 275-280.

Received: 13 December 2017

Accepted: 21 December 2017

Correspondence to: Assist. Karmen Stanič, M.D., Ph.D., Institute of Oncology Ljubljana, Zaloška 2, SI-1000 Ljubljana, Slovenia. Phone: +386 15879 502; Fax: +386 15879 400; E-mail: kstanic@onko-i.si

Disclosure: No potential conflicts of interest were disclosed.

Background. Postmastectomy radiotherapy (PMRT) improves survival by eliminating potential occult lesions in the chest wall and lymphatic drainage area. Meta-analysis has shown that PMRT reduces mortality and local recurrence of patients with node positive breast cancer, but there is no specific data about the effectiveness of PMRT in a subgroup of patients with a high number of positive axillary lymph nodes (PALN). The aim of the study was to analyse the impact of the number of PALN on local and distant metastasis occurrence, overall survival (OS) and distant metastases free survival (DMFS) in patients treated with PMRT.

Patients and methods. We reviewed medical records of 129 consecutive breast cancer patients with PALN, treated at Institute of Oncology Ljubljana with PMRT between January 2003 and December 2004. We grouped patients according to the number of PALN as follows: Group 1 (less than 15 PALN) and Group 2 with more than 15 PALN. All patients received adjuvant systemic therapy according to the clinical guidelines. We analysed number of locoregional (LR) recurrences, distant metastasis, overall survival (OS), progression free survival (PFS) and DMFS.

Results. After the median follow-up time of 11.5 years, the Kaplan-Meier survival analysis of PALN showed significantly shorter OS $(p=0.006)$, shorter PFS $(p=0.002)$ and shorter DMFS $(p<0.001)$ in the group of $>15$ PALN. Only one LR was found in the group of patients with more than 15 PALN. In multivariate analysis more than 15 PALN and treatment with anthracycline chemotherapy statistically significantly influenced OS and DMFS. For PFS presence of more than 15 PALN were the only independent factor of shorter survival.

Conclusions. Patients with more than 15 PALN have shorter DMFS, PFS and OS as compared to patients with less than 15 PALN, though they receive the same LR treatment. More studies with higher number of patients included are needed to further evaluate our findings.

Key words: breast cancer; postmastectomy radiotherapy; positive axillary lymph nodes

\section{Introduction}

The aim of postmastectomy radiotherapy (PMRT) in breast cancer patients is to improve loco-regional (LR) control and survival by eliminating potential occult lesions in the chest wall and lymphatic drainage area. These benefits have been consistently reported in multiple studies..$^{1-3}$ Meta-analysis, made by Early Breast Cancer Trialists' Collaborative
Group (EBCTCG), published in Lancet in 2014, has shown that PMRT reduces recurrence and breast cancer mortality in women with one to three positive lymph nodes. ${ }^{4}$ There is almost no doubt that the group of breast cancer patients with more than 3 positive axillary lymph nodes (PALN) benefit from PMRT, but it is questionable if the benefit is the same all over the described group. ${ }^{2}$ Currently, inadequate data exist to provide answer to this question. 
According to our clinical experience, we assumed that patients with many PALN might have a greater chance of already present micrometastatic disease and therefore a greater chance for distant spread than for LR relapse. However, existing TNM classification with 10 lymph nodes as the lower limit of the group with the highest number of PALN might not correspond appropriately to or clinical experiences as it seems to be set too low. ${ }^{5}$ Majority of breast cancer patients treated with mastectomy receive systemic therapy within the frame of radical treatment, with impact also on potential subclinical LR lesions, destroying them before they become clinically evident and symptomatic. Moreover, at the time of eventual distant spread, patients receive another line of systemic treatment, which again impact also on potential subclinical LR lesions.

Even though all patients in the studied group received the same kind of LR treatment, we anticipated that patients with many PALN would have less LR relapses than group of patients with smaller number of PALN. We also hypothesized that patients with many PALN might have shorter overall survival and distant metastasis free survival.

In addition to proven benefits, radiation therapy (RT) also has its known side effects. Those need to be over weighted with a benefit of the treatment. Among long known toxicities of RT are skin changes, secondary tumours and lately highly reported

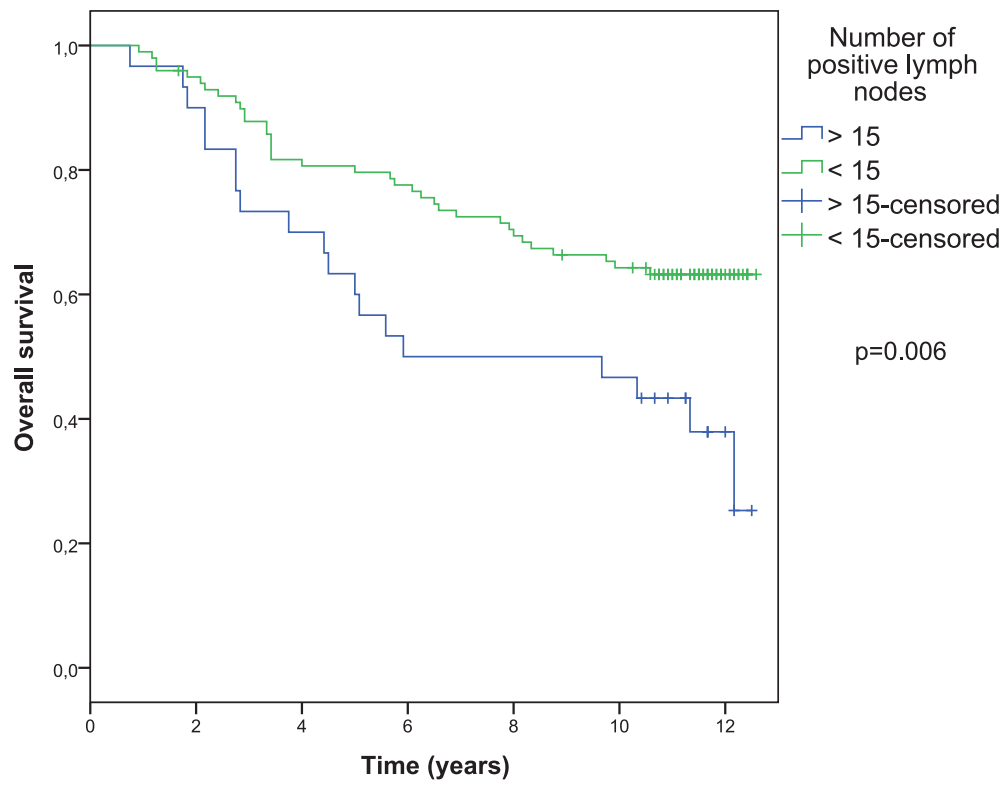

FIGURE 1. Overall survival according to number of positive axillary lymph nodes. cardio-toxic effects. ${ }^{6-9}$ In multimodality treatment specific toxicities of each treatment are potentiated, therefore benefit of RT in patients with many PALN should be addressed.

\section{Patients and methods}

We reviewed medical records of 129 consecutive breast cancer patients with PALN who were treated at the Institute of Oncology Ljubljana with PMRT between January 2003 and December 2004. All the patients received RT to the thoracic wall and ipsilateral periclavicular region according to clinical guidelines. External beam irradiation was delivered with photons and/or electrons with a total dose of $48 \mathrm{~Gy}-56 \mathrm{~Gy}$ in 5 daily fractions per week.

We grouped patients according to the number of PALN in groups with a low and that with a high number of PALN. Our clinical experiences suggest that the lower limit for N3 class in TNM classification is set too low to reliably predict a greater chance of a distant recurrence. ${ }^{5}$

Therefore, we performed the following grouping for local recurrence: Group 1 (1-3 PALN); Group 2 (4-15 PALN); Group 3 (more than 15 PALN). In further analysis we compared only patients with more than 15 (Group 1) to less than 15 PALN (Group 2).

All patients received adjuvant systemic therapy according to the clinical guidelines. At that time treatment with trastuzumab has not been a part of standard adjuvant treatment yet. However, HER2 was determined in all patients. Additional variables were age, tumour histology, tumour grade, tumour size, estrogen receptor (ER) status, progesterone receptor status (PR), lympho-vascular invasion, peri-neural invasion, adjuvant hormonal therapy, adjuvant chemotherapy and intrinsic subtypes. Breast cancer subtypes were defined based on 2015 St. Gallen Consensus Conference classification but without information on $\mathrm{Ki}-67$, as routine testing was not available at that time, as follows: Luminal A (ER positive, HER2 negative, PR $>20 \%$ positive), luminal B (ER positive, HER2 positive or negative, $\mathrm{PR}<20 \%$ ), HER2-overexpression (HER2 positive, ER negative, $\mathrm{PR}$ negative), triple negative breast cancer (TNBC) or basal like (ER negative, PR negative, HER2 negative). ${ }^{10}$

Data was analysed with respect to overall survival (OS), progression free survival (PFS) distant metastasis free survival (DMFS) and locoregional free survival (LRFS). 


\section{Statistical analysis and ethical consideration}

OS time was calculated from the date of surgery to the date of death or last follow up. PFS time was calculated from the date of surgery to the time of first progression, either locoregional or distant. LRFS was calculated from the date of surgery to the first LR progression and DMFS to the event of first distant metastasis. Kaplan-Meier methods were used to estimate survival curves. Log rank tests were used for univariate analysis to compare the survival contribution. In multivariate Cox regression only variables with $p<0.2$ from univariate analysis were included. Data was calculated using SPSS v.20 statistical package. All p values reported were based on the two-sided hypothesis and were considered statistical significant for values $<0.05$ and $95 \%$ confidence interval (CI) of hazard ratio (HR) that did not include 1.

The study has been approved by Institutional Review Board Committee and Ethics Committee and conducted in accordance with the declaration of Helsinki.

\section{Results}

The median follow-up time was 11.5 years. Patients' characteristics are presented in Table 1. Median age of breast cancer patients was 56 years and majority had invasive ductal carcinoma $(80.7 \%)$. Beside to mastectomy, patients also had lymph node dissection. Mean number of examined lymph nodes was 19 (SD 8.1). With respect to breast cancer subtype Luminal A was predominant (47.3\%), followed by luminal B (31\%), HER2 group (12.4\%) and TNBC $(9.3 \%)$. HER2 positive patients were present in 28.7\%. All ER positive patients (77.5\%) received hormonal therapy (HT). Adjuvant chemotherapy was delivered to $83 \%$ of patients.

Kaplan-Meier survival analysis for lymph node groups with > 15 PALN (30 patients) vs. $<15$ PALN (99 patients) showed significant difference in median OS survival. For the group with $>15$ PALN was 5.9 years (SD 3.6), while for the group with $<15$ PALN median time was not reached at the time of analysis $(p=0.006)$ as shown on Figure 1 . The number of LR recurrences occurred as follows: 5 (17\%) in group with 1-3 PALN, 4 (5.7\%) in group 4-15 PALN and the lowest number was in the group with the highest number of PALN-Group 3; only 1 recurrence (3.3\%). Further analysis was not performed due to low number of events. Distant me-
TABLE 1. Patients' characteristics

\begin{tabular}{|c|c|c|}
\hline Patients characteristics & No patients & $\%$ \\
\hline & 129 & 100 \\
\hline \multicolumn{3}{|l|}{ Age (years) } \\
\hline Median & 56 & \\
\hline Q1-Q3 & $48-64$ & \\
\hline \multicolumn{3}{|l|}{ Histology } \\
\hline IDC & 104 & 80.7 \\
\hline ILC & 25 & 19.3 \\
\hline \multicolumn{3}{|l|}{ Tumour size } \\
\hline $\mathrm{Tl}$ & 29 & 9.3 \\
\hline $\mathrm{T} 2$ & 79 & 45.7 \\
\hline T3 & 21 & 45.0 \\
\hline \multicolumn{3}{|l|}{ Histological grade } \\
\hline Gl & 12 & 9.3 \\
\hline G2 & 59 & 45.7 \\
\hline G3 & 58 & 45.0 \\
\hline \multicolumn{3}{|l|}{ Lymphovascular invasion } \\
\hline Yes & 54 & 41.9 \\
\hline No & 64 & 49.6 \\
\hline N/A & 11 & 8.5 \\
\hline \multicolumn{3}{|l|}{ Perineural invasion } \\
\hline Yes & 20 & 15.5 \\
\hline No & 83 & 64.3 \\
\hline N/A & 26 & 20.2 \\
\hline \multicolumn{3}{|l|}{ No. of positive axillary lymph nodes (PALN) } \\
\hline $1-3$ & 29 & 22.5 \\
\hline $4-15$ & 70 & 54.3 \\
\hline$>15$ & 30 & 23.2 \\
\hline \multicolumn{3}{|l|}{ No. of PALN according to $\mathrm{N}$ category } \\
\hline $\mathrm{N} 1$ & 29 & 22.5 \\
\hline N2 & 47 & 36.5 \\
\hline N3 & 53 & 41.0 \\
\hline \multicolumn{3}{|l|}{ Estrogen receptor } \\
\hline Positive & 100 & 77.5 \\
\hline Negative & 29 & 22.5 \\
\hline \multicolumn{3}{|l|}{ Progesteron receptor } \\
\hline Positive & 85 & 65.9 \\
\hline Negative & 44 & 34.1 \\
\hline \multicolumn{3}{|l|}{ HER-2 overexpression } \\
\hline Positive & 37 & 28.7 \\
\hline Negative & 92 & 71.3 \\
\hline \multicolumn{3}{|l|}{ Adjuvant hormone therapy } \\
\hline Yes & 101 & 78.3 \\
\hline No & 28 & 21.7 \\
\hline \multicolumn{3}{|l|}{ Adjuvant chemotherapy } \\
\hline Yes & 108 & 83.7 \\
\hline No & 21 & 16.3 \\
\hline \multicolumn{3}{|l|}{ Adjuvant chemotherapy with anthracyclines } \\
\hline Yes & 99 & 76.7 \\
\hline No & 30 & 23.2 \\
\hline \multicolumn{3}{|l|}{ Breast cancer subtype } \\
\hline Luminal A & 61 & 47.3 \\
\hline Luminal B & 40 & 31.0 \\
\hline Her2-overexpression & 16 & 12.4 \\
\hline Triple negative breast cancer (TNBC) & 12 & 9.3 \\
\hline
\end{tabular}




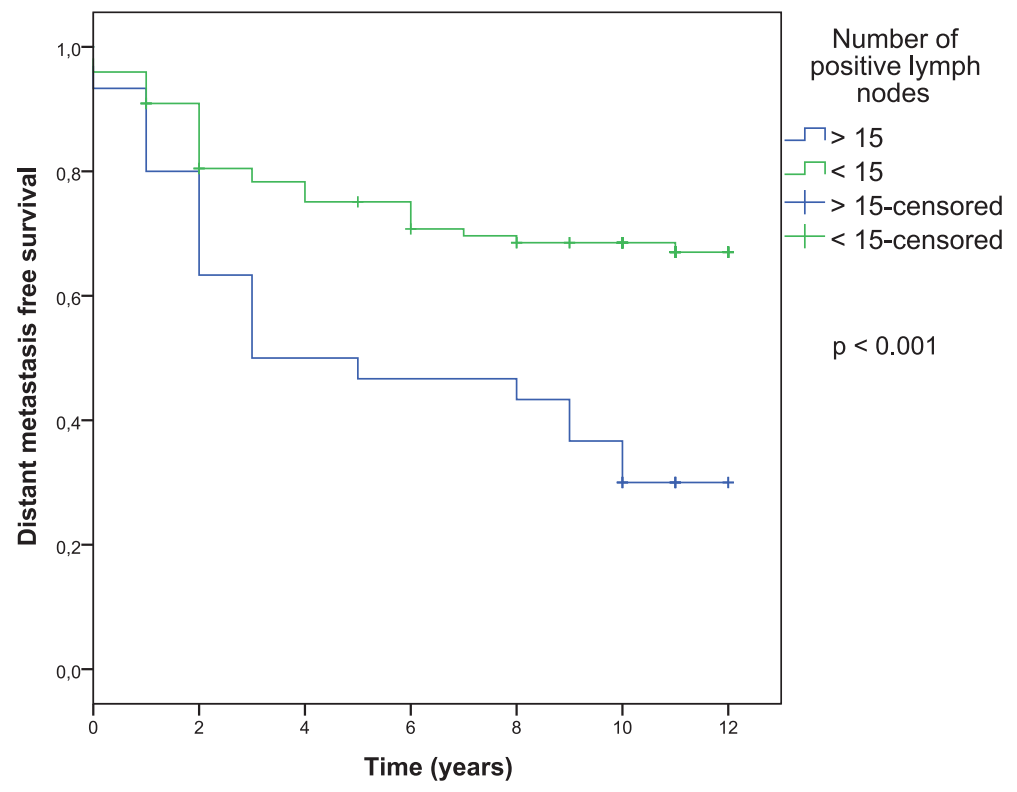

FIGURE 2. Distant metastasis free survival according to number of positive axillary lymph nodes.

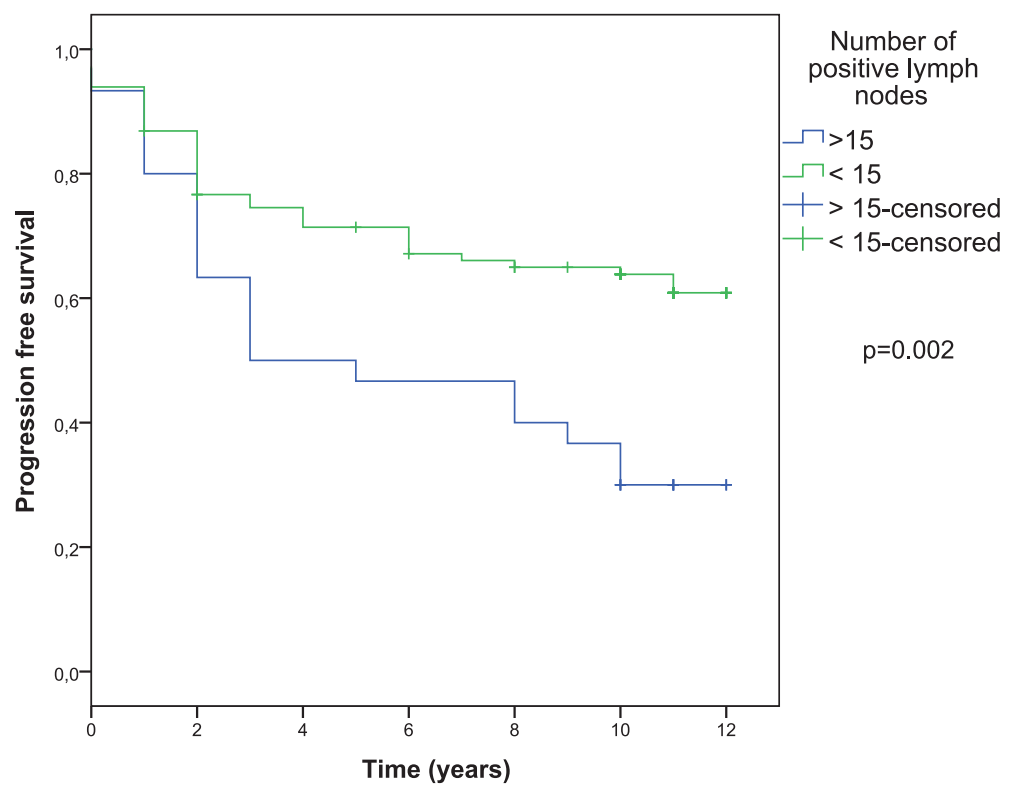

FIGURE 3. Progression free survival according to number of positive axillary lymph nodes.
Multivariate analysis showed two variables with significant influence on OS, adjuvant chemotherapy with anthracyclines $(\mathrm{p}=0.005, \mathrm{HR}=0.39$, CI $0.20-0.75$ ) and group of patients with more than 15 positive PALN ( $\mathrm{p}=0.002$, HR $=2.52$, CI 1.38-4.57). For PFS only more than 15 PALN showed significant influence $(\mathrm{p}=0.003, \mathrm{HR}=2.24)$. None of the investigated factors independently influenced LRF. Adjuvant chemotherapy with anthracyclines $(\mathrm{HR}=0.51)$ and more than 15 PALN (HR 3.05) were also statistically significant for DMFS. We found no significant influence of the breast cancer subtypes on any of the categories analysed.

\section{Discussion}

Patients with more than 15 PALN had shorter PFS, OS and DMFS. On the other hand, treatment with anthracyclines showed increased DMFS and OS. In our analysis only one patient experienced LR in the group with more than 15 PALN.

Published data provides evidence that the most significant prognostic factor for OS in patients with early-stage breast cancer is the presence or absence of axillary lymph node involvement. Furthermore, there is a direct relationship between the number of involved axillary nodes and the risk for distant recurrence. ${ }^{11,12}$ More than three decades ago Fisher et al. reported that the 5-year OS for patients with node-negative disease was $82.8 \%$ compared with $73 \%$ for $1-3$ positive nodes, $45.7 \%$ for $4-12$ positive nodes, and $28.4 \%$ for $\geq 13$ positive nodes. ${ }^{13}$ Nowadays we use more potential systemic therapy and there are substantial improvements in OS of patients with clinically localized breast cancer. ${ }^{14}$ But in spite of better systemic treatment, the number of PALN remains a negative prognostic factor for breast cancer patients.

The impact of local therapy on survival of patients with breast cancer has been debated for decades. In breast cancer, three theories of cancer spread in breast cancer exist. ${ }^{15}$ More than hundred years ago dr. Wiliam Halsted believed that breast cancer begins as a strictly local disease and that tumour cells only spread haematogenously to other organs at a later stage. ${ }^{16}$ Unfortunately, only $12 \%$ of patients treated with radical mastectomy, survived 10 years. The poor outcome with the Halstedian approach, as well as the observation that $20 \%-30 \%$ of node-negative patients ultimately develop metastatic disease, led to the "systemic view « theory, proposed by dr. Bernard Fisher. ${ }^{17,18}$ He believed that breast cancer is systemic disease and if distant 
metastases were destined to develop, such metastases already exist at the time of diagnosis of the breast cancer.

Neither Halstedian nor the systemic view could have explained the course of the disease for all breast cancer. ${ }^{15}$ A third hypothesis, the "spectrum" theory, synthesized aspects of both opposing approaches. As Punglia et al. wrote in their article, »this theory holds that for many breast cancers there is a time when tumor cells have not metastasized to distant sites but that it is generally not known whether this time has passed at the point of diagnosis for any patient «. They also wrote that this theory acknowledges that the greater the likelihood that systemic spread has occurred at the time of diagnosis in a patient, the lower the likelihood that local therapy will influence the patient's survival. ${ }^{15}$ The results of our analysis are in accordance with their theory showing that patients with higher number of PALN have low number of LR recurrences and greater likelihood for shorter OS. ${ }^{11,12,13}$ With regards to the spectrum theory and explanation of Punglia et al., it seems that in the subgroup of patients with many PALN local treatment has a minor impact.

Punglia et al. also pointed out, that contribution of improved LR control to survival depends on the effectiveness of systemic treatment. ${ }^{15}$ Later on, Poortmans added to this factor also the component of metastatic risk of primary tumour and he concluded that with combining both components, the contribution of improved LR treatment to the final outcome can be estimated. ${ }^{19}$ We agree that metastatic potential of different breast cancer tumours is very heterogenic, as is disease itself. It is well known that different breast cancer subtypes have different OS and in our opinion this is the component that really needs to be explored..$^{20}$ However, in our analysis we didn't show any significant difference in breast cancer subgroups regarding PALN and OS, probably due to small number of patients in each subgroup.

Based on our analysis, it seems that our results fit best to the spectrum theory. We grouped patients according to the number of PALN in groups with a low and that with a high number of PALN. Interestingly, Fisher in his article grouped patients very similar as we did. ${ }^{13}$ It seems that if the number of PALN is very high, the chance of haematogenic relapse at distant sites is higher than the chance of lymphogenic local relapse and the time to evident haematogenic distant metastases is shorter than the time to local lymphogenic relapse which is in line with the theory of Punglia and collegues. ${ }^{15}$
Finally, it is also in concordance with the results of our multivariate analysis, showing that adjuvant chemotherapy with antracyclines and more than 15 PALN predict for shorter DMFS.

Our study was limited with a small number of patients. According to clinical guidelines, all patients with a high number of PALN are treated with PMRT; therefore, it was not possible to compare studied patients with a cohort treated without RT. For the future, trials comparing our results with a cohort of patients treated also with adjuvant trastuzumab would be interesting. Trastuzumab, with proven important impact on overall survival, became a part of adjuvant treatment in 2005, soon after our cohort was treated..$^{21}$ We assume that it might further diminish a need for PMRT in HER2 positive breast cancer patients due to its very potent systemic effectiveness.

\section{Conclusions}

Patients with more than 15 PALN have shorter OS, PFS and DMFS compared to patients with less than 15 PALN though they receive the same LR therapy. They also have the smallest number of LR recurrences. Our results suggest that systemic treatment with anthracyclines is important component of adjuvant treatment for patients with higher number of PALN and radiation treatment might be questionable in this group, but studies with larger number of patients would be needed to answer this question.

\section{References}

1. Danish Breast Cancer Cooperative Group, Nielsen HM, Overgaard M, Grau C, Jensen AR, Overgaard J. Study of failure pattern among high-risk breast cancer patients with or without postmastectomy radiotherapy in addition to adjuvant systemic therapy: long-term results from the Danish Breast Cancer Cooperative Group DBCG 82 b and c randomized studies. J Clin Oncol 2006; 24: 2268-75. doi: 10.1200/JCO.2005.02.8738

2. Clarke M, Collins R, Darby S, Davies C, Elphinstone P, Evans V, et al. Effects of radiotherapy and of differences in the extent of surgery for early breast cancer on local recurrence and 15-year survival: an overview of the randomised trials. Lancet 2005; 366: 2087-106. doi: 10.1016/S0140-6736(05)67887-7

3. Ragaz J, Olivotto IA, Spinelli JJ, Phillips N, Jackson SM, Wilson KS, et al. Locoregional radiation therapy in patients with high-risk breast cancer receiving adjuvant chemotherapy: 20-year results of the British Columbia randomized trial. J Nat/ Cancer Inst 2005; 97: 116-26. doi: 10.1093/jnci/djh297

4. EBCTCG (Early Breast Cancer Trialists' Collaborative Group). Effect of radiotherapy after mastectomy and axillary surgery on 10-year recurrence and 20-year breast cancer mortality: meta-analysis of individual patient data for 8135 women in 22 randomised trials. Lancet 2014; 383: 2127-35. doi: 10.1016/S0140-6736(14)60488-8

5. Coates AS, Winer EP, Goldhirsch A, Gelber RD, Gnant M, Piccart-Gebhart $\mathrm{M}$, et al. Tailoring therapies - improving the management of early breast cancer: St Gallen International Expert Consensus on the Primary Therapy of Early Breast Cancer 2015. Ann Oncol 2015; 26: 1533-46. doi: 10.1093/ annonc/mdv221 
6. Darby SC, McGale P, Taylor CW, Peto R. Long-term mortality from heart disease and lung cancer after radiotherapy for early breast cancer: prospective cohort study of about 300,000 women in US SEER cancer registries. Lancet Oncol 2005; 6: 557-65. doi: 10.1016/S1470-2045(05)70251-5

7. Darby SC, Ewertz M, McGale P, Bennet AM, Blom-Goldman U, Brønnum D, et al. Risk of ischemic heart disease in women after radiotherapy for breast cancer. N Engl J Med 2013; 368: 987-98. doi: 10.1056/NEJMoa1209825

8. Marinko T, Borstnar S, Blagus R, Dolenc J, Bilban-Jakopin C. Early cardiotoxicity after adjuvant concomitant treatment with radiotherapy and trastuzumab in patients with breast cancer. Radiol Oncol 2018; 52: 204-212. doi: 10.2478/raon-2018-0011

9. Sardar P, Kundu A, Chatterjee S, Nohria A, Nairooz R, Bangalore S, et al. Long-term cardiovascular mortality after radiotherapy for breast cancer: a systematic review and meta-analysis. Clin Cardiol 2017; 40: 73-81. doi: 10.1002/clc. 22631

10. Union Against Cancer (UICC). TNM classification of malignant tumours Sobin LH, Gospodarowicz MK, Wittekind C, editors. 7th edition. Chichester, UK: Wiley-Blackwell; 2009.

11. Saez RA, McGuire WL, Clark GM. Prognostic factors in breast cancer. Semin Surg Oncol 1989; 5: 102-10.

12. Nemoto T, Natarajan N, Bedwani R, Vana J, Murphy GP. Breast cancer in the medial half. Results of 1978 National Survey of the American College of Surgeons. Cancer 1983; 51: 1333-8. doi: 10.1002/1097-0142(19830415) 51:8<1333::AID-CNCR2820510802>3.0.CO;2-T

13. Fisher $B$, Bauer $M$, Wickerham $D L$, Redmond $C K$, Fisher $E R$, Cruz $A B$, et al. Relation of number of positive axillary nodes to the prognosis of patients with primary breast cancer. An NSABP update. Cancer 1983; 52: 1551-7. doi: 10.1002/1097-0142(19831101)52:9<1551::AIDCNCR2820520902>3.0.CO;2-3

14. Early Breast Cancer Trialists' Collaborative Group (EBCTCG). Effects of chemotherapy and hormonal therapy for early breast cancer on recurrence and 15-year survival: an overview of the randomised trials. Lancet 2005; 365: 1687-717. doi: 10.1016/S0140-6736(05)66544-0

15. Punglia RS, Morrow M, Winer EP, Harris JR. Local therapy and survival in breast cancer. N Engl J Med 2007; 356: 2399-405. doi: 10.1056/ NEJMra065241

16. Halsted WS. I. The results of radical operations for the cure of carcinoma of the breast. Ann Surg 1907; 46: 1-19. doi: 10.1097/SLA.0b013e31824b7e35

17. Fisher B, Gebhardt MC. The evolution of breast cancer surgery: past, present, and future. Semin Oncol 1978; 5: 385-94.

18. Cianfrocca M, Goldstein $\amalg$. Prognostic and predictive factors in earlystage breast cancer. Oncologist 2004; 9: 606-16. doi: 10.1634/theoncologist.9-6-606

19. Poortmans P. Postmastectomy radiation in breast cancer with one to three involved lymph nodes: ending the debate. Lancet 2014; 383: 2104-6. doi: 10.1016/S0140-6736(14)60192-6

20. Haque R, Ahmed SA, Inzhakova G, Shi J, Avila C, Polikoff J, et al. Impact of breast cancer subtypes and treatment on survival: an analysis spanning two decades. Cancer Epidemiol Biomarkers Prev 2012; 21: 1848-55. doi: 10.1158/1055-9965.EPI-12-0474

21. Cameron D, Piccart-Gebhart MJ, Gelber RD, Procter M, Goldhirsch A de Azambuja $E$, et al. 11 years' follow-up of trastuzumab after adjuvant chemotherapy in HER2-positive early breast cancer: final analysis of the HERceptin Adjuvant (HERA) trial. Lancet 2017; 389: 1195-205. doi: 10.1016/ S0140-6736(16)32616-2 